\title{
Suppressed and enhanced tunneling ionization of transition-metal atoms and cations: A time-dependent density-functional-theory study on nickel
}

\author{
$\mathrm{Xi} \mathrm{Chu}(1)$ \\ Department of Chemistry and Biochemistry, The University of Montana, Missoula, Montana 59812, USA \\ Gerrit C. Groenenboom $\odot$ \\ Institute for Molecules and Materials, Radboud University Nijmegen, Heyendaalseweg 135, 6525 AJ Nijmegen, The Netherlands
}

(Received 24 January 2020; accepted 6 April 2020; published 29 April 2020)

\begin{abstract}
We study the tunneling ionization (TI) of $\mathrm{Ni}, \mathrm{Ni}^{+}$, and $\mathrm{Ni}^{2+}$ with a time-dependent density-functional-theory method and reproduce the puzzling suppression of the $\mathrm{TI}$ of $\mathrm{Ni}$ and $\mathrm{Ni}^{+}$and the enhancement of $\mathrm{TI}$ in $\mathrm{Ni}^{2+}$. Numerical results reveal that for all three species the electron tunnels from a $4 s$ orbital; that is, excitation precedes tunneling for both of the cations, for which the highest orbitals are $3 d$. The effective radial potentials for the $d$ orbitals have a centrifugal barrier, while there is no such barrier for the $s$ orbitals. At the classical turning point for the $3 d$ orbital, the $3 d$ to $4 s$ excitation energy is lower than the centrifugal potential for the $d$ orbitals. Two factors of opposite nature are identified in this work. On the one hand, electrons moving away from the nucleus in the intense laser fields induce an attractive potential that effectively lowers the energy level and thus suppresses tunneling. Excitation, on the other hand, has the opposite effect and enhances tunneling. The energy gap between $4 s$ and $3 d$ is small for $\mathrm{Ni}^{+}$and therefore suppression wins. As the charge of the cation increases, the excitation energy becomes much greater, and for $\mathrm{Ni}^{2+}$ enhancement dominates. Based on a similar analysis, we expect enhanced TI for several transition-metal cations of charge 2 and higher.
\end{abstract}

DOI: 10.1103/PhysRevA.101.043423

\section{INTRODUCTION}

Tunneling ionization (TI) is a strong-field process in which a bound electron tunnels to a free-electron state [1]. The electron may recombine or rescatter, which is utilized in attosecond imaging and spectroscopy [2-5]. The precise description of tunneling ionization is key to these new technologies.

An intense IR field may be treated as quasistatic because of the combination of a large field strength and a relative small field frequency. As such, the ionization is a tunneling process often modeled by Ammosov-Delone-Krainov (ADK) theory [6]. As a single active electron (SAE) theory, ADK predicts the TI rates of inert gases reasonably well [7], while it overestimates those rates for some other atoms and molecules [8-10].

Transition-metal atoms and their ions in different oxidation states form coordination complexes of chemical importance. In short-wavelength IR lasers, the reduction of the ionization yields for the transition-metal atoms $\mathrm{V}, \mathrm{Ni}, \mathrm{Pd}, \mathrm{Ta}$, and $\mathrm{Nb}$ is equivalent to an increase of the ionization potential by a few eV [10,11]. Multielectron effects are considered the cause of the discrepancy [12-15]. Another study on the TI of transition-metal ions obtained from metallocenes revealed an interesting trend: while the ionization rates of the neutral atoms $\mathrm{Cr}, \mathrm{Fe}, \mathrm{Ni}$, and $\mathrm{Ru}$ and their singly charged cations are lower than the ADK predictions, those of the doubly charged cations are higher, which leads to considerably lower saturation intensities than the ADK values [16]. The question then arises as to why ADK theory underestimates the TI rates of certain oxidation states of a transition metal while it overestimates others. Naturally, the explanation lies in the approximations made in ADK theory.

A time-dependent density-functional-theory (TDDFT) study reproduced the measured ionization yields of vanadium atoms and explained the apparent suppression when compared to ADK predictions [15]. Unlike the ADK approach, the TDDFT method does not assume a quasistatic picture but instead solves the time propagation of a set of Kohn-Sham (KS) orbitals. It includes dynamic many-electron interactions. It describes how much the ground state is changed in an intense laser field, which is incurred not only by the direct Coulomb interaction between the active electron and the field but also by the changed many-electron interaction induced by the field as well. The study revealed an induced attractive potential near the nucleus, which is caused by the electron density moving away, lowering the ground-state energy and hence effectively increasing the ionization potential. It also showed that momentary accumulation and depletion of the electron density of one side of the nucleus versus the other elevates the barrier for tunneling and thus reduces the ionization rate.

One would expect that both many-electron effects, which lower the TI rates, affect the cations as well. Why are the TIs of some cations enhanced? The answer, we postulate, should lie in the different electronic structures of the cations. Another TDDFT study demonstrated the difference that the electronic structure can make in TI by comparing the ground state of vanadium $\left[\left(3 d^{3} 4 s^{2}\right)^{4} F\right]$ to the $\left(3 d^{4} 4 s\right)^{6} D$ excited state. Even though the ionization potential of the ${ }^{4} F$ quartet state is higher than that of the ${ }^{6} D$ sextet state, the TI rates of the sextet turned 
out to be lower because of exchange blockade for the higher spin state [17].

As far as the electronic structures are concerned, an obvious observation is that the highest electron of all the neutral transition-metal atoms mentioned above, whose TI rates are lower than the ADK rates, occupies an $s$ orbital. The highest electrons of all the cations that exhibit enhanced ionization, however, are in $d$ orbitals. To the best of our knowledge, TI from a $d$ shell is not yet a target of substantial research. Projecting the time-dependent wave function of the highest occupied spin orbital of $\mathrm{Ni}^{+}$and of $\mathrm{Ni}^{2+}$ for $r \geqslant 7 a_{0}$ to the field-free orbitals, we find that in the tunneling regime projections to the $4 s$, rather than to the $3 d$ orbitals, dominate. This finding suggests an excitation-followed-by-TI mechanism for these ions.

The centrifugal barrier associated with angular momentum quantum number $l$,

$$
k_{l}(r)=\frac{l(l+1) \hbar^{2}}{2 r^{2}},
$$

where $r$ is the distance of the electron from the nucleus, is not considered in ADK tunneling theory. For $d$ orbitals $(l=$ 2 ), however, it is substantial in the short range, i.e., when $r$ is small. For a cation, the electron density of an occupied $d$ orbital is concentrated at a short range, where the excitation energy from a $3 d$ to an unoccupied $4 s$ orbital is far less than the centrifugal potential energy.

We choose to study the TI of nickel because both Smits et al. [10] and Murakami et al. [16] measured the ionization yields of it. While the values given by Murakami et al. are in arbitrary units, those by Smits et al. are not, so these are good benchmarks for our calculations. The conclusions of this study may be applicable to a class of transition metals.

We adopt an all-electron TDDFT method for treating openshell atoms. This approach was implemented for the neutralatom vanadium and explained its suppressed TI [15,17]. It is an optimized effective potential (OEP) formalism, which correctly accounts for the long-range exchange-correlation potential. The method is briefly reviewed in Sec. II.

Modeling of ionization is given in Sec. III. In Sec. IV we discuss the orbital energies from the initial-state calculations. The computed ionization probabilities are given in Sec. V. In Sec. VI we analyze the induced potential caused by manyelectron effects, and in Sec. VII we identify the mechanism that explains the suppression and enhancement of TI. Finally, in Sec. VIII we conclude.

\section{TIME-DEPENDENT KOHN-SHAM EQUATIONS WITH AN OPTIMIZED EFFECTIVE POTENTIAL FOR TRANSITION-METAL ATOMS AND IONS}

For a TDDFT calculation it is necessary that the initial state is representable by a Slater determinant, for which we have to specify the $n, l, m$, and $m_{s}$ quantum numbers for each spin orbital. To study an open-shell system, one has the choice of a spin-restricted or a spin-unrestricted treatment. While spinrestricted DFT may be adopted to determine the ionization potential as the energy difference between the species after and prior to ionization, we prefer spin-unrestricted TDDFT to calculate the ionization rate.
TABLE I. Calculated orbital energies $\epsilon$ (in $\mathrm{eV}$ ) of nickel $\mathrm{Ni}^{c+}$ with $c=0,1$, and 2, compared to the measured ionization potentials $\left(I_{p}\right)$ from Ref. [21]. Conf. = configuration.

\begin{tabular}{|c|c|c|c|c|c|c|c|}
\hline \multirow[b]{2}{*}{$c$} & \multicolumn{2}{|c|}{$\mathrm{Ni}^{c+}$} & \multicolumn{2}{|c|}{$\mathrm{Ni}^{(c+1)+}$} & \multirow[b]{2}{*}{$I_{p}$} & \multirow[b]{2}{*}{ Orbital } & \multirow[b]{2}{*}{$-\epsilon$} \\
\hline & Level & Conf. & Level & Conf. & & & \\
\hline \multirow[t]{4}{*}{0} & ${ }^{3} F_{4}$ & $3 d^{8} 4 s^{2}$ & ${ }^{4} F_{9}$ & $3 d^{8}\left({ }^{3} F\right) 4 s$ & 8.68 & $4 s_{\downarrow}$ & 8.48 \\
\hline & & & ${ }^{2} F_{7}^{2}$ & $3 d^{8}\left({ }^{3} F\right) 4 s$ & 9.32 & $4 s_{\uparrow}$ & 8.78 \\
\hline & & & ${ }^{4} F_{9}^{2}$ & $3 d^{7} 4 s^{2}$ & 13.97 & $3 d_{\downarrow}$ & 12.74 \\
\hline & & & ${ }^{2} G_{\frac{9}{2}}^{2}$ & $3 d^{7} 4 s^{2}$ & 16.36 & $3 d_{\uparrow}$ & 14.93 \\
\hline \multirow[t]{2}{*}{1} & ${ }^{2} D_{\frac{5}{5}}$ & $3 d^{9}$ & ${ }^{3} F_{4}$ & $3 d^{8}$ & 18.17 & $3 d_{\downarrow}$ & 19.52 \\
\hline & & & ${ }^{1} G_{4}$ & $3 d^{8}$ & 21.03 & $3 d_{\uparrow}$ & 22.11 \\
\hline \multirow[t]{2}{*}{2} & ${ }^{3} F_{4}$ & $3 d^{8}$ & ${ }^{4} F_{\frac{9}{2}}$ & $3 d^{7}$ & 35.19 & $3 d_{\downarrow}$ & 37.44 \\
\hline & & & ${ }^{2} G_{\frac{9}{2}},{ }^{2} H_{9}$ & $3 d^{7}$ & 37.64 & $3 d_{\uparrow}$ & 39.47 \\
\hline
\end{tabular}

We start by expressing the initial $N$-electron wave functions of $\mathrm{Ni}, \mathrm{Ni}^{+}$, and $\mathrm{Ni}^{2+}$ as Slater determinants of the occupied spin orbitals of the ground state,

$$
\Psi\left(t=t_{0}\right)=\frac{1}{\sqrt{N !}} \operatorname{det}\left[\phi_{1 \sigma_{1}} \phi_{2 \sigma_{2}} \cdots \phi_{N \sigma_{N}}\right],
$$

where $\sigma_{i}=\alpha$ or $\beta$ is the spin of the $i$ th electron and $t_{0}$ is the initial time. We include all the electrons in the calculation, i.e., $N=28$ for $\mathrm{Ni}$ and $N=26$ for $\mathrm{Ni}^{2+}$. The valence-electron configurations are given in Table I. The field-free spin orbitals satisfy the static KS equation

$$
\hat{H}_{\sigma}^{(0)}(\boldsymbol{r}) \phi_{j \sigma}(\boldsymbol{r})=\epsilon_{j \sigma} \phi_{j \sigma}(\boldsymbol{r}), \quad j=1,2, \ldots, N_{\sigma},
$$

where $j$ is the orbital index, $N_{\sigma}$ is the number of electrons for the $\sigma$ spin, and $\boldsymbol{r}$ is the electron coordinate.

The time-dependent KS equations are Euler equations resulting from the quantum action [18,19]

$$
A[\Psi]=\int_{t_{0}}^{t_{1}} d t\left\langle\Psi(\boldsymbol{r}, t)\left|i \frac{\partial}{\partial t}-\hat{H}(t)\right| \Psi(\boldsymbol{r}, t)\right\rangle
$$

having a stationary point at the time-dependent density $\rho(\boldsymbol{r}, t)$. The time-dependent $N$-electron wave function is

$$
\Psi(\boldsymbol{r}, t)=\frac{1}{\sqrt{N !}} \operatorname{det}\left[\psi_{1 \sigma_{1}}(\boldsymbol{r}, t) \psi_{2 \sigma_{2}}(\boldsymbol{r}, t) \cdots \psi_{N \sigma_{N}}(\boldsymbol{r}, t)\right] .
$$

The density at coordinate $\boldsymbol{r}$ and time $t$ is

$$
\rho(\boldsymbol{r}, t)=\rho_{\alpha}(\boldsymbol{r}, t)+\rho_{\beta}(\boldsymbol{r}, t),
$$

where

$$
\rho_{\sigma}(\boldsymbol{r}, t)=\sum_{i=1}^{N_{\sigma}} \psi_{i \sigma}^{*}(\boldsymbol{r}, t) \psi_{i \sigma}(\boldsymbol{r}, t) .
$$

In an intense laser field, the time-dependent $\mathrm{KS}$ equation is

$$
i \hbar \frac{\partial}{\partial t} \psi_{j \sigma}(\boldsymbol{r}, t)=\left[\hat{H}_{\sigma}^{(0)}(\boldsymbol{r})+\Delta v_{\sigma}(\boldsymbol{r}, t)-\boldsymbol{E}(t) \cdot \boldsymbol{r}\right] \psi_{j \sigma}(\boldsymbol{r}, t),
$$

where $\boldsymbol{E}$ is the electric field of the laser and $\Delta v_{\sigma}$ is the induced potential.

Projecting Eq. (5) onto a determinant of field free orbitals, we find contributions of single, double, triple, and higher excitations. Such multiple excitations and deexcitations are 
many-electron by nature and they give rise to the change in electron density

$$
\Delta \rho(\boldsymbol{r}, t)=\sum_{\sigma=\alpha}^{\beta} \rho_{\sigma}(\boldsymbol{r}, t)-\rho_{\sigma}\left(\boldsymbol{r}, t_{0}\right),
$$

which in turn creates the induced potential

$$
\Delta v_{\sigma}(\boldsymbol{r}, t)=\iiint \frac{\Delta \rho\left(\boldsymbol{r}^{\prime}, t\right)}{\left|\boldsymbol{r}-\boldsymbol{r}^{\prime}\right|} d^{3} \boldsymbol{r}^{\prime}+\Delta V_{\mathrm{xc}, \sigma}(\boldsymbol{r}, t) .
$$

The change in the exchange-correlation potential is given by

$$
\Delta V_{\mathrm{xc}, \sigma}(\boldsymbol{r}, t)=V_{\mathrm{xc}, \sigma}(\boldsymbol{r}, t)-V_{\mathrm{xc}, \sigma}\left(\boldsymbol{r}, t_{0}\right),
$$

and the time-dependent exchange-correlation potential is expressed as

$$
V_{\mathrm{xc}, \sigma}(\boldsymbol{r}, t)=-\frac{1}{\rho_{\sigma}(\mathbf{r}, t)} \sum_{i} \sum_{j} \operatorname{Re}\left[\psi_{j \sigma}^{*}(\boldsymbol{r}, t) \psi_{i \sigma}(\boldsymbol{r}, t) \iiint \frac{\psi_{i \sigma}^{*}\left(\boldsymbol{r}^{\prime}, t\right) \psi_{j \sigma}\left(\boldsymbol{r}^{\prime}, t\right)}{\left|\mathbf{r}-\mathbf{r}^{\prime}\right|} d^{3} \boldsymbol{r}^{\prime}\right] .
$$

\section{MODELING THE TUNNELING IONIZATION OF NICKEL ATOMS AND ITS CATIONS}

The initial state is set to be one of the $2 J+1$ degenerate states of a level defined by quantum numbers $L, S$, and $J$. The $J$ quantum numbers of the nickel atom and ions are listed in Table I as subscripts of the term symbols. When we use the spin-unrestricted treatment, the two states with $M_{J}= \pm J$ are representable by a single determinant. Ionization probabilities are identical for the two.

For the neutral nickel atom and its cations in Table I, the only partially filled subshell is $3 d$. Degenerate states with different $M_{J}$ values involve occupation of sets of $3 d$ orbitals of various $m$ quantum numbers. The projection of the angular momentum on the $z$ axis is $m \hbar$, the polarization direction of the electric field $\boldsymbol{E}$ is along the $z$ axis, and

$$
E(t) \equiv|\boldsymbol{E}(t)|=f(t) \sin \omega t,
$$

where $\omega$ is the angular frequency of the incident light and $f(t)$ is the field strength. Among all $3 d$ orbitals, tunneling ionization from the one with $m=0$ has by far the greatest rates. All the degenerate ground states of the neutral atom and cations listed in Table I have the $3 d_{0}$ orbital occupied; therefore, all the degenerate ground states of the same species of nickel share similar ionization rates, and the TI rate of the state with $M_{J}=J$ is representative of the ground-state TI rate.

To be consistent with the experiment of Smits et al. [10], we set the wavelength to $1500 \mathrm{~nm}$. A constant field strength $f(t)=F$ for $90 \mathrm{fs}$ is assumed in the experiment. Since the initial Hamiltonian does not incorporate any external field, we let $f(t)$ linearly increase from 0 to $F$ in 10 fs (four optical cycles).

All the occupied spin orbitals are propagated in time from $t=-10$ to $90 \mathrm{fs}$. The set of spin orbitals at $t=0$ is renormalized. We calculate the survival probability for each spin orbital as

$$
n_{i \sigma}=\iiint \psi_{i \sigma}^{*}(\boldsymbol{r}, T) \psi_{i \sigma}(\boldsymbol{r}, T) d^{3} \mathbf{r}
$$

where $T=90 \mathrm{fs}$, and the ionization probability for each spin orbital as

$$
\gamma_{i \sigma}=1-n_{i \sigma}
$$

The ionization probability is calculated as the sum

$$
P=\sum_{\sigma} \sum_{i=1}^{N_{\sigma}} \gamma_{i \sigma}
$$

We implemented a generalized pseudospectral method as described in [20] to solve the time-dependent equations. This method features an unevenly distributed grid that is denser towards the nucleus and a split operator representation for the time evolution. We set the maximum radial distance from the nucleus to be $r_{\max }=300 a_{0}$ and place an absorbing boundary at $r_{0}=40 a_{0}$ such that for $r$ between $r_{0}$ and $r_{\max }$ the wave function is multiplied by a factor of $\cos ^{0.25}[\pi(r-$ $\left.\left.r_{0}\right) / 2\left(r_{\max }-r_{0}\right)\right]$ at every time step.

\section{INITIAL STATES DETERMINED BY DFT/OEP CALCULATIONS}

In this TDDFT study of $\mathrm{TI}$ of $\mathrm{Ni}, \mathrm{Ni}^{+}$, and $\mathrm{Ni}^{2+}$, we first calculate the initial state of the atom and ions with an OEP formalism. As expected from an OEP calculation, minus the $4 s$-orbital energy of the neutral atom matches the measured ionization potential. The highest occupied spin orbital of $\mathrm{Ni}$ is $4 s_{\downarrow}$. For $3 d$ orbitals of all three species, however, there exists a discrepancy that increases with the charge of the ion. Nonobservance of Koopmans's theorem is a reflection that the energy and charge distribution of the $3 d$ orbitals change significantly as the ion charge increases, which bears significance for tunneling ionization.

The negatives of the $4 s$ and $3 d$ spin-orbital energies of $\mathrm{Ni}$, $\mathrm{Ni}^{+}$, and $\mathrm{Ni}^{2+}$ from the static DFT calculations are given in the column labeled $-\epsilon$ in Table I. We also quote the measured ionization potentials for removing an electron from those orbitals while keeping other electrons in the same spin orbitals. The level and configuration before and after ionization are both listed.

Because the $3 d$ subshell is not filled, $\hat{H}_{\sigma}^{(0)}$ is not spherical, which removes the degeneracy of the $3 d_{0}, 3 d_{ \pm 1}$, and $3 d_{ \pm 2}$ orbitals. The orbital energy differences between various $3 d$ orbitals are within $0.3 \mathrm{eV}$ for the three species we study. In Table I we give the $-\epsilon$ value for $3 d_{0}$ because it is the most active $3 d$ orbital due to its orientation. The orientation effect influences TI much more than an orbital energy difference of $0.3 \mathrm{eV}$ or less. 
According to Koopmans's theorem, the negative of the orbital energy obtained by a spin-restricted Hartree-Fock calculation of a closed-shell atom is equal to its ionization potential. As our systems are open-shell atoms and ions and the spin orbitals, $3 d$ in particular, do not remain frozen when an electron is removed, Koopmans's theorem does not necessarily apply. Nevertheless, we tabulate both $I_{p}$ and $-\epsilon$ because $I_{p}$ is what we input into ADK calculations presented in Sec. V and $\epsilon$ is taken as the energy level of the SAE in the qualitative analysis offered in Sec. VII.

The ground state of the nickel atom has 15 spin-up electrons $(\sigma=\alpha)$ and 13 spin-down electrons $(\sigma=\beta)$. Spin orbitals $3 d_{\uparrow}$ are filled with five electrons. The only partially filled spin orbitals are $3 d_{\downarrow}$. The magnetic quantum numbers $m$ for the three occupied ones are 0,1 , and 2, respectively. Removal of the highest electron from $4 s_{\downarrow}$ results in level $\left[3 d^{8}\left({ }^{3} F\right) 4 s\right]^{4} F_{\frac{9}{2}}$ of $\mathrm{Ni}^{+}$, which is not the $\left(3 d^{9}\right)^{2} D_{\frac{5}{2}}$ ground state of the cation. Ionization of neutral nickel in the ground state to the singly charged cation in the ground state takes $7.64 \mathrm{eV}$ [21]. The excitation energy of the cation is an additional $1.02 \mathrm{eV}$. The calculated $-\epsilon_{4 s_{\downarrow}}$ is very close to the measured $I_{p}$.

It takes more energy to remove an electron from $4 s_{\uparrow}$. The absolute value of the orbital energy deviates from the corresponding $I_{p}$ by $-0.54 \mathrm{eV}$. With an electron in $3 d_{\downarrow}$ or $3 d_{\uparrow}(m=0)$ removed, the cation is in an even higher state. Koopmans's theorem becomes less applicable here: $-\epsilon_{3 d_{\downarrow}}$ and $-\epsilon_{3 d_{\uparrow}}$ are more than an $\mathrm{eV}$ less than $I_{p}$. This is because the value of $\epsilon_{4 s}-\epsilon_{3 d}$ is increased in the cation and the ionization potential includes this increase of $\epsilon_{4 s}$ in addition to the increase in $-\epsilon_{3 d}$.

The $4 s$ orbitals of $\mathrm{Ni}^{+}$and $\mathrm{Ni}^{2+}$ are not occupied. The calculated $-\epsilon_{3 d}$ is 1 or $2 \mathrm{eV}$ more than $I_{p}$. This is due to the decrease of $\epsilon_{3 d}$ after ionization caused by reduced electronelectron repulsion and increased electron-nucleus attraction. The change in all the occupied orbitals accompanying ionization, including those that cause the breakdown of Koopmans's theorem, is included in our TDDFT formalism in the format of the time-dependent induced potential $\Delta v_{\sigma}$, which in turn determines the time-dependent orbitals. Therefore, the TDDFT calculations produce reasonable TI results in spite of the difference between $I_{p}$ and $-\epsilon$.

\section{COMPUTED IONIZATION PROBABILITIES}

In Fig. 1 we plot the calculated ionization probabilities of $\mathrm{Ni}, \mathrm{Ni}^{+}$, and $\mathrm{Ni}^{2+}$ obtained by all-electron TDDFT calculations as black, red, and green crosses, respectively. We show only calculated probabilities up to 0.4 . If probabilities are higher than that, the system deviates too much from the initial state and may not be represented well by TDDFT. Also shown in are ADK values (the dashed lines) and the experimentally measured relative yields for ionizing $\mathrm{Ni}$ (the circles).

The ionization probabilities are plotted on linear and $\log$ scales in the top and bottom panels, respectively. The logscale plot enhances the resolution for lower ionization probabilities at the relatively lower laser intensities. Ionization is a multiphoton rather than tunneling process when the laser intensity is low enough. In this regime the TDDFT calculations
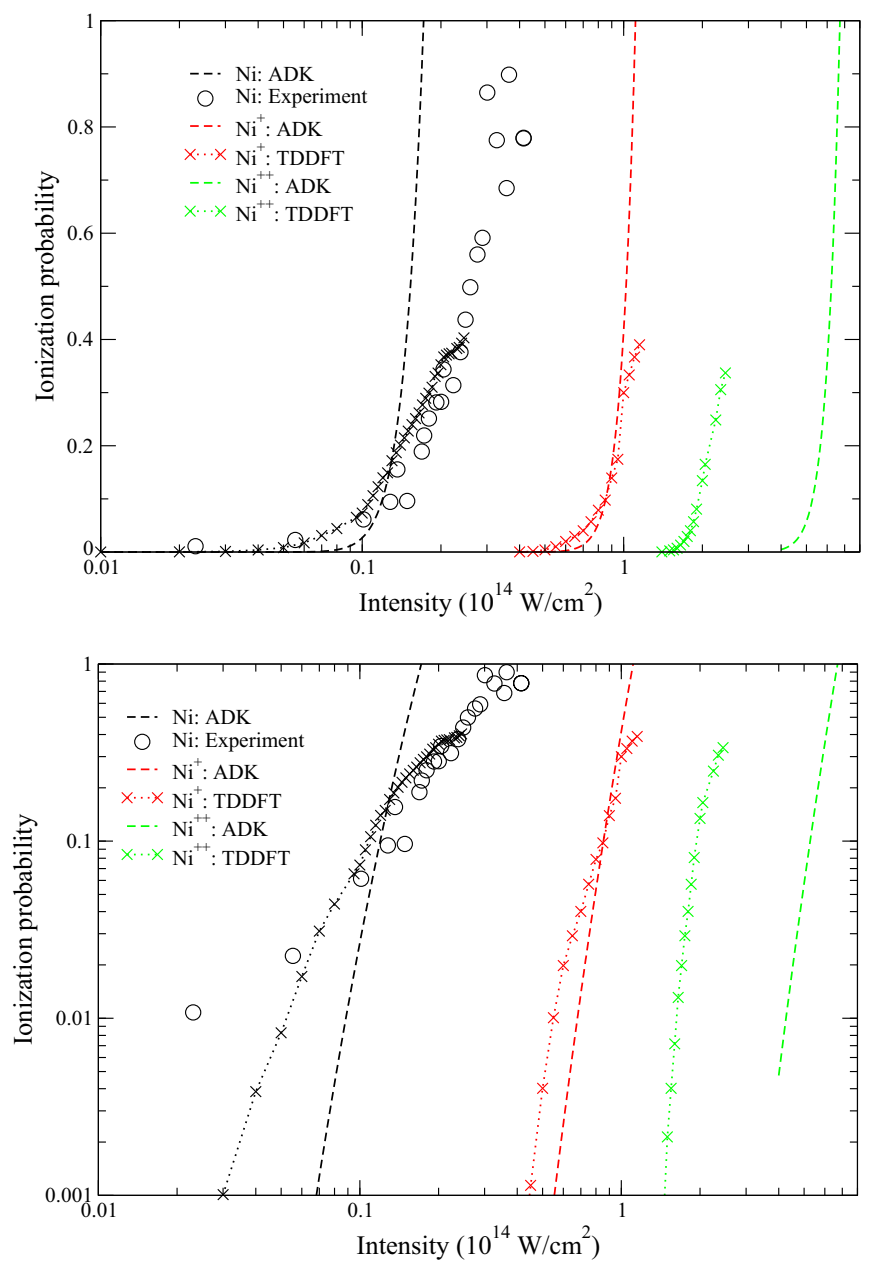

FIG. 1. Ionization probabilities of a neutral nickel atom and cations of nickel in 1500-nm, 90-fs laser pulses. The probabilities are presented in both the linear scale (top) and log scale (bottom). The experimental values are reproduced from a linearly scaled figure in Ref. [10]. Limited by the resolution of the print in [10], the probability reading near 0.01 may be far off the original value.

give higher ionization rates than the ADK model. In modeling ionization of transition-metal atoms in the multiphoton regime, electron correlation becomes increasingly important at lower laser intensities. A local exchange-correlation functional without memory as in our method may not be sufficient. In this paper we focus our discussion on tunneling ionization, which occurs at relatively higher laser intensities when the Keldysh parameter is less than 1 [1].

For the neutral atom, ionization probabilities calculated by TDDFT agree well with the experimental values [10]. For $I>$ $1.3 \times 10^{13} \mathrm{~W} / \mathrm{cm}^{2}$, probabilities from TDDFT calculations are much lower than the ADK results.

For the singly charged cation, $\mathrm{Ni}^{+}$, the TDDFT results are lower than those from the ADK calculations for the higher laser intensities and higher for the lower intensities. Contrasting the substantial differences between the TDDFT and ADK results for $\mathrm{Ni}$, the two are much closer for $\mathrm{Ni}^{+}$. According to Murakami et al. [16], the saturation intensity for ionizing $\mathrm{Ni}^{+}$is slightly higher than the prediction of ADK, 

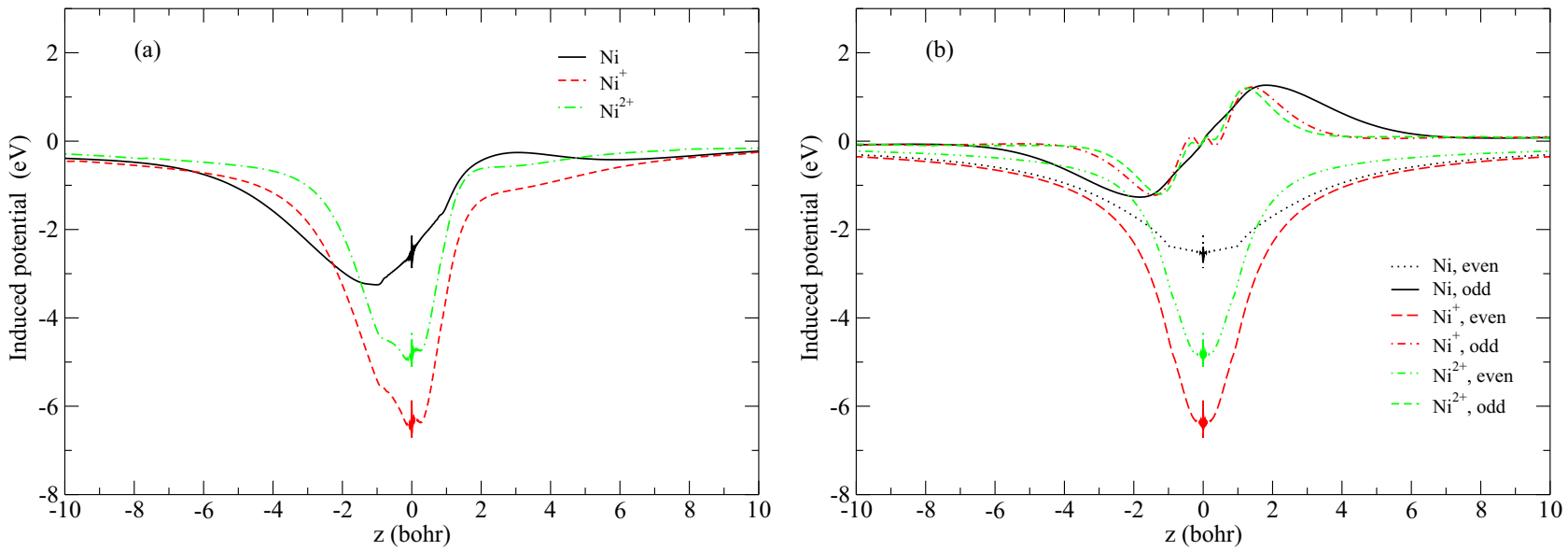

FIG. 2. The induced potential $\Delta v_{\downarrow}$ of $\mathrm{Ni}$ in $2 \times 10^{13} \mathrm{~W} / \mathrm{cm}^{2}$, of $\mathrm{Ni}^{+}$in $10^{14} \mathrm{~W} / \mathrm{cm}^{2}$, and of $\mathrm{Ni}^{2+}$ in $2.35 \times 10^{14} \mathrm{~W} / \mathrm{cm}^{2}, 1500-\mathrm{nm}$ lasers . The potential is obtained by solving the TDDFT equations for a time near $45 \mathrm{fs}$, when $E(t)=F$.

and the tunneling ionization yields are a little lower than the ADK values, which is consistent with our findings.

The most striking data in Fig. 1 are the huge disagreement between TDDFT and ADK for $\mathrm{Ni}^{2+}$. The pattern is reversed in this case, and $\mathrm{TI}$ of $\mathrm{Ni}^{2+}$ is substantially enhanced relative to the ADK picture, which is consistent with Murakami et al. as well [16].

As far as the TI rates relative to the ADK values are concerned, the TDDFT calculations reproduce the experimental results. Therefore, we analyze the TDDFT data to explain the apparent suppression of tunneling ionization in $\mathrm{Ni}$ and $\mathrm{Ni}^{+}$ and enhancement in $\mathrm{Ni}^{2+}$.

\section{ANALYSIS OF MANY-ELECTRON DYNAMICS}

Many-electron dynamics, which is excluded by ADK theory, gives rise to the induced potential defined in Eq. (10). Without any external field, an induced core can have extraordinary effects on the electron [22]. Here, we analyze and compare the induced potentials in intense laser fields for $\mathrm{Ni}$, $\mathrm{Ni}^{+}$, and $\mathrm{Ni}^{2+}$, which are shown in Fig. 2(a) to reveal the cause of "suppressed" and "enhanced" ionization. The highest electrons are all spin down; we therefore plot $\Delta v_{\downarrow}$ at a time when the electric field is maximized.

We choose the intensities of $2 \times 10^{13} \mathrm{~W} / \mathrm{cm}^{2}$ for $\mathrm{Ni}$, $10^{14} \mathrm{~W} / \mathrm{cm}^{2}$ for $\mathrm{Ni}^{+}$, and $2.35 \times 10^{14} \mathrm{~W} / \mathrm{cm}^{2}$ for $\mathrm{Ni}^{2+}$. Because the calculated ionization probabilities for the three species are similar (Fig. 1), at the long range for $z \geqslant 10 a_{0}$ all three species converge to a similar value.

Driven by an intense laser field, electrons move away from the nucleus, and hence, an attractive potential is induced at the short range, which is shown for all three species in Fig. 2(a). Attraction turns out to be dominant in the entire range, which suggests the cause of suppressed TI.

The oscillations of $\Delta v_{\downarrow}$ near the origin indicate that the electron density moves into or out of $s$ orbitals, which have nodes near the nucleus. The $4 s$ orbitals are not occupied for the cations; however, the involvement of high $s$ orbitals suggests the possibility of excitation to $4 s$ prior to tunneling ionization, which enhances tunneling due to reduced barrier height and width.
Excitations are tractable in our computation. When solving the TDDFT equation, we compute the projection of the timedependent spin orbital $\psi_{i \sigma}$ onto the spherical harmonics $Y_{l, m}$ [20] at every time step,

$$
g_{i \sigma, l, m}(r, t)=\int_{0}^{2 \pi} \int_{0}^{\pi} Y_{l, m}^{*}(\theta, \phi) \psi_{i \sigma}(r, \theta, \phi, t) \sin \theta d \theta d \phi,
$$

where $r, \theta$, and $\phi$ are spherical polar coordinates. Initially, there is only one combination of $l$ and $m$ that has nonzero projection for each spin orbital. For example, when $i \sigma$ stands for $3 d_{0, \downarrow}$ of a cation, the only nonzero projection is $g_{3 d_{0, \downarrow}, 2,0}\left(r, t_{0}\right)$ for $t=t_{0}$. When $t=t_{m}$, i.e., when the electric field is maximized, $\left|g_{3 d_{0, \downarrow}, 0,0}\left(r>7 a_{0}, t_{m}\right)\right|^{2}$ dominates the population at the long range for both $\mathrm{Ni}^{+}$in the $2 \times 10^{13} \mathrm{~W} / \mathrm{cm}^{2}$ laser and $\mathrm{Ni}^{2+}$ in the $10^{14} \mathrm{~W} / \mathrm{cm}^{2}$ laser. It demonstrates that the highest electron in $3 d_{0 \downarrow}$ is excited to $4 s_{\downarrow}$, from which it tunnels.

In Fig. 2(b) we further separate $\Delta v_{\downarrow}$ into functions odd and even in the $z$ coordinate, i.e.,

$$
\Delta v_{\downarrow}(\boldsymbol{r}, t)=\Delta v_{\downarrow}^{\text {even }}(\boldsymbol{r}, t)+\Delta v_{\downarrow}^{\text {odd }}(\boldsymbol{r}, t) .
$$

The even function is attractive, and the odd function contributes to the barrier of tunneling. Polarization is the leading term of the induced odd potential. The polarizability decreases considerably from Ni to $\mathrm{Ni}^{+}$and drops even further for $\mathrm{Ni}^{2+}$. With the increasing laser intensity we chose for the three systems, the three barriers shown in Fig. 2(b) are of similar heights.

The even potentials are mainly due to excitations to the unoccupied $s$ orbitals, whereas the odd potentials are due to $p$ orbitals. The amplitudes of the odd potentials are less than those of the even potentials, and hence, the induced potentials are mostly attractive. Accordingly, excitation to the $s$ orbitals appear to be much more substantial.

Collectively, $4 s$ electrons are further away from the nucleus than the $3 d$ electrons; therefore, a $4 s$ electron moving outward induces less attraction than a $3 d$ electron moving outward, which explains the smallest magnitude for $\mathrm{Ni}$ for the short range. Between $\mathrm{Ni}^{+}$and $\mathrm{Ni}^{2+}$, the higher charge makes it more difficult for a $3 d$ electron of $\mathrm{Ni}^{2+}$ to move outward than 

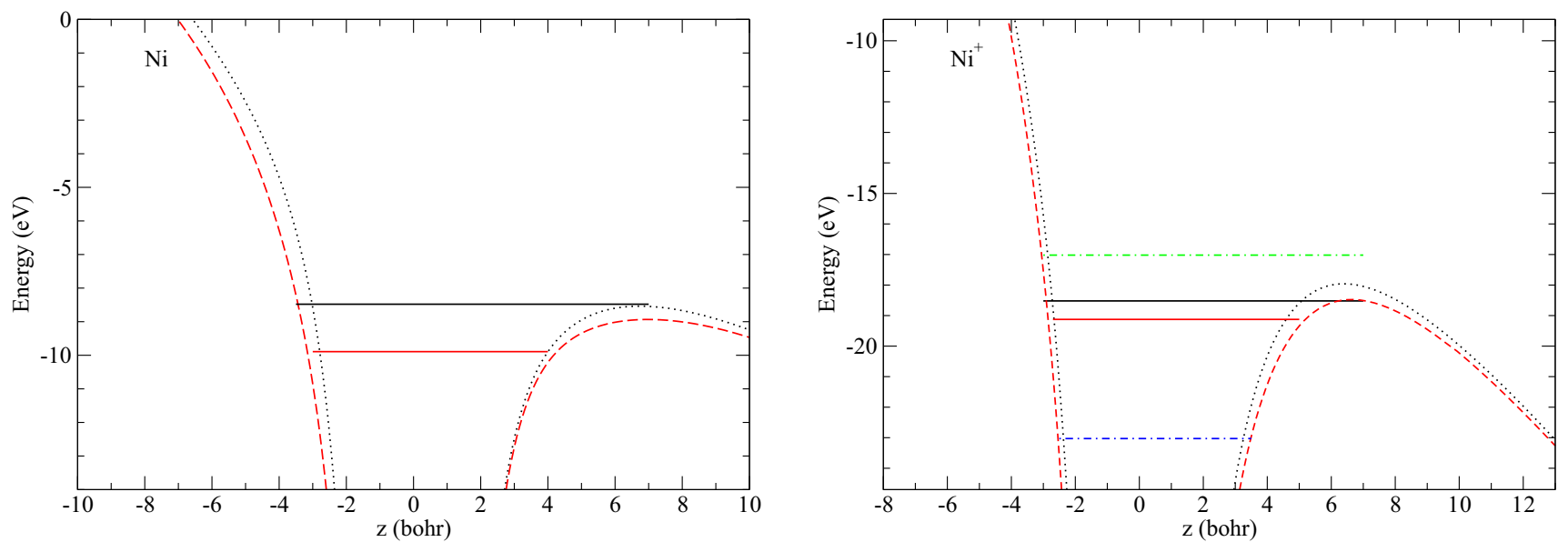

FIG. 3. Energy diagram for $\mathrm{Ni}$ in a $1500-\mathrm{nm}$ laser of $2 \times 10^{13} \mathrm{~W} / \mathrm{cm}^{2}$ (left) and for $\mathrm{Ni}^{+}$in a $1500-\mathrm{nm} \mathrm{laser} \mathrm{of} 10^{14} \mathrm{~W} / \mathrm{cm}^{2}($ right). Solid black line: the field-free energy level for the highest occupied spin orbital. Black dotted line: the potential of the electric field at the peak intensity plus the field-free electronic potential. Red solid line: the shifted energy level of $4 s_{\downarrow}$ near $t=45$ fs when the electric field is maximized. Red dashed line: the TDDFT electronic potential at the same time. Green dot-dashed line: the unshifted and unoccupied $4 s_{\downarrow}$ of $\mathrm{Ni}^{+}$. Blue dot-dashed line: the shifted $3 d_{\downarrow}$ of $\mathrm{Ni}^{+}$at the same time.

that of $\mathrm{Ni}^{+}$, which explains the smaller magnitude for $\mathrm{Ni}^{2+}$ for the short range.

\section{SUPPRESSED TUNNELING AND ENHANCED TUNNELING}

Ionization suppression or enhancement is relative to a SAE picture, in which the electron-ion interaction is assumed to be unaffected by the external field. The electron tunnels from the highest occupied spin orbital and is subject to the potential of the electric field plus the electron-ion potential,

$v_{\downarrow}^{s}(\boldsymbol{r}, t)=-\boldsymbol{E}(t) \cdot \boldsymbol{r}-\frac{Z e}{r}+\iiint \frac{\rho\left(\boldsymbol{r}^{\prime}\right)}{\left|\boldsymbol{r}-\boldsymbol{r}^{\prime}\right|} d^{3} \boldsymbol{r}^{\prime}+\Delta V_{\mathrm{xc}, \downarrow}(\boldsymbol{r})$,

where $Z=28$ is the nuclear charge.

What is additional in the TDDFT description is the induced potential defined in Eq. (10) and depicted in Fig. 2,

$$
v_{\downarrow}(\boldsymbol{r}, t)=v_{\downarrow}^{s}(\boldsymbol{r}, t)+\Delta v_{\downarrow}(\boldsymbol{r}, t) .
$$

Because of $\Delta v_{\downarrow}(\boldsymbol{r}, t)$, the energy of the highest electron is time dependent as well and is shifted from the static orbital energy.

In Figs. 3 and 4, we plot the energy and potential of the highest electron of $\mathrm{Ni} \mathrm{Ni}^{+}$, and $\mathrm{Ni}^{2+}$, respectively. The SAE model results are in black, and the TDDFT results are in red. The comparison presented in these figures shows how $\Delta v$ alters both the energy level and the barrier, which in turn influences the tunneling rate.

The potentials and shifted energies depend on time. The result in Figs. 3 and 4 are for a time near $t=45$ fs and for $\sin \omega t=1$; that is, $E(t)$ is maximized. The time-dependent energy shift is different from the AC Stark shift with a leading term of $-\frac{\alpha(\omega) F^{2}}{4}$, where $\alpha$ is the dynamic polarizability. Polarization contributes to only the odd function component of $\Delta v_{\sigma}$. However, as we see in Fig. 2(b), the even function component is more substantial and makes a greater contribution to the energy shift in the tunneling regime.

In the left panel of Fig. 3 we present the data for a neutral nickel atom, for which the highest spin orbital is $4 s_{\downarrow}$. The intensity of the laser is $2 \times 10^{13} \mathrm{~W} / \mathrm{cm}^{2}$, as in Fig. 2. In the SAE picture (black), it is over-the-barrier ionization, and hence, the ionization rate is high. In the TDDFT picture (red) the $4 s_{\downarrow}$ energy level drops by $1.2 \mathrm{eV}$ under the influence of $\Delta v_{\downarrow}$. Since $\Delta v_{\downarrow}$ is more attractive near the nucleus, the downshift of the barrier at $6 a_{0}$ is less. As such, the tunneling is through the barrier, and therefore, it is suppressed relative to the SAE depiction of over-the-barrier ionization. This comparison demonstrates that the suppressed $\mathrm{TI}$ of $\mathrm{Ni}$ is because of the

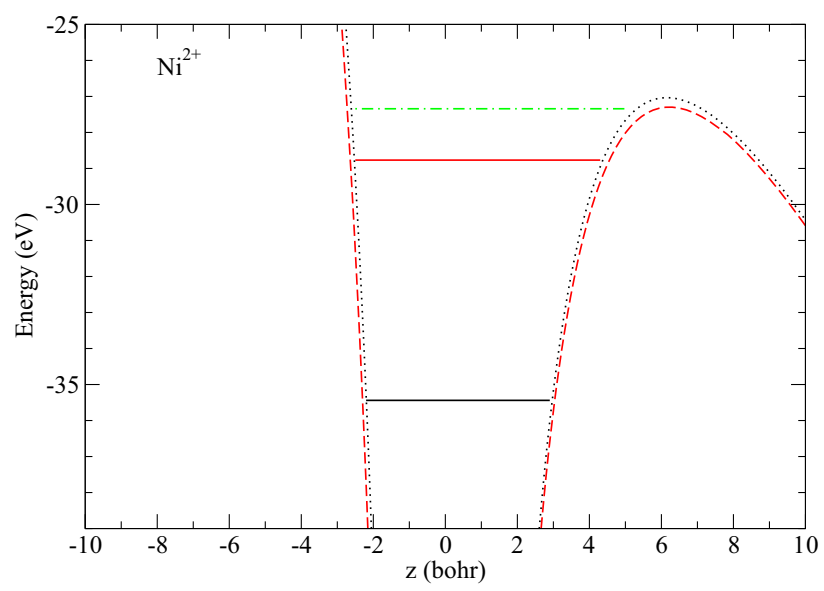

FIG. 4. Energy diagram for $\mathrm{Ni}^{2+}$ in a 1500 -nm laser of $2.35 \times$ $10^{13} \mathrm{~W} / \mathrm{cm}^{2}$. Solid black line: the field-free energy level for the highest occupied spin orbital, $3 d_{\downarrow}$. Black dotted line: the potential of the electric field at the peak intensity plus the field-free electronic potential. Red solid line: the shifted energy level of $4 s_{\downarrow}$ at the moment when the electric field is maximized. Red dashed line: the TDDFT calculated electronic potential at the peak intensity. Green dot-dashed line: the unshifted and unoccupied $4 s_{\downarrow}$ of $\mathrm{Ni}^{2+}$. 
laser-induced attractive potential caused by the outgoing of the electrons.

Similar information on $\mathrm{Ni}^{+}$is shown in the right panel of Fig. 3. From the SAE point of view (black), ionization is tunneling from the unshifted $3 d_{\downarrow}$ orbital, and the potential is given in Eq. (19) with a laser intensity of $10^{14} \mathrm{~W} / \mathrm{cm}^{2}$. For comparison, we illustrate the TDDFT description in red, which is tunneling from the shifted $4 s_{\downarrow}$ orbital through a barrier lowered by the induced potential $\Delta v_{\downarrow}$. Initially, the $4 s_{\downarrow}$ orbital is unoccupied. In a laser of $10^{14} \mathrm{~W} / \mathrm{cm}^{2}$, however, $4 s_{\downarrow}$ is populated substantially at the long range and the shifted $4 s_{\downarrow}$-orbital energy is lower than the unshifted energy of the $3 d_{\downarrow}$ orbital by $0.6 \mathrm{eV}$. As a reference, we also plot the unshifted $4 s_{\downarrow}$ and shifted $3 d_{\downarrow}$ as green and blue dashdotted lines, respectively. It turns out that the SAE picture in black and the TDDFT picture in red involve a similar barrier height. The ionization probability is calculated to be 0.4169 by ADK theory and 0.2999 by our TDDFT method. This comparison is consistent with the experimental observation that the TI rates of $\mathrm{Ni}^{+}$are only slightly lower than the predictions of the ADK theory [16].

In Fig. 4, TI of $\mathrm{Ni}^{2+}$ described by TDDFT (red) and that in the SAE description (black) are drastically different. Tunneling is substantially enhanced over the SAE description, which matches the difference in TDDFT vs ADK values shown in Fig. 1.

Similar to the case of $\mathrm{Ni}^{+}$, the highest occupied spin orbital of $\mathrm{Ni}^{2+}$ is $3 d_{\downarrow}$, but the electron tunnels from the $4 s_{\downarrow}$ orbital in the intense field. In Fig. 4 the shifted energy of the $4 s_{\downarrow}$ orbital is $6.7 \mathrm{eV}$ higher than the energy of the unshifted $3 d_{\downarrow}$ orbital, which causes significant enhancement of tunneling and which also explains the trend of TI enhancement of transition-metal cations of charge 2 (yielding cations of charge 3 ) [16].

As can be seen from Figs. 3 and 4, the classical turning point moves to shorter radial distances with higher charge. At the short range, the centrifugal potential for a $d$ orbital is particularly high. This potential is not incorporated in the ADK expression but is part of $\hat{H}^{(0)}$ in our TDDFT formalism, which keeps the electrons occupying $3 d$ orbitals from moving outwards. Even though the excitation energy from $3 d_{\downarrow}$ to $4 s_{\downarrow}$ increases with the charge of the cation, it remains lower than the centrifugal potential between nuclei and the classical turning point. The $4 s$ orbitals have no centrifugal potential to prevent the outgoing of the wave function.

Figures 3 and 4 are used to demonstrate suppressed or enhanced TI, while specific ionization rates and probabilities depend on solutions of the TDDFT equation, Eq. (8). Equation (13) shows that the photon frequency appears in the TDDFT equation; therefore, the calculated ionization probabilities depend on the wavelength of the incident laser. A TDDFT study on the strong-field ionization of $\mathrm{H}_{2}$ showed that the ionization rate increases with shorter wavelengths [23]. How much it increases depends on the specific electronic structure and the laser intensity. For $\mathrm{H}_{2}$ the rate in a 1500-nm laser is about 1.1 times the static field limit. In a 780-nm laser, the ionization rate is 4.0 times the limit [23]. Since the electronic structures of the three species we study here are all substantially different from that of $\mathrm{H}_{2}$, we cannot use the data of $\mathrm{H}_{2}$ to predict how their ionization rates depend on the wavelength. Specific calculations are needed instead. However, we expect a suppression or enhancement trend similar to those shown in Figs. 3 and 4 for a laser with shorter wavelength as well, although both the shifts of the barrier and the shifts of the energy levels will be different, which leads to different tunneling rates.

\section{SUMMARY AND CONCLUSIONS}

This is a TDDFT study of $\mathrm{TI}$ of $\mathrm{Ni}, \mathrm{Ni}^{+}$, and $\mathrm{Ni}^{2+}$. Like in other transition metals, the $3 d$-orbital energy is higher than that of $4 s$ for the neutral atom but lower for the cations. Thus, the highest occupied orbital is $4 s$ for the neutral atom and $3 d$ for the cation. While the charge of a cation increases in the process of ionization, the $4 s-3 d$ energy gap increases, the $3 d$ orbitals get closer to the nucleus, and their energies become lower as well.

The calculated ionization probabilities for $\mathrm{Ni}$ agree with the experimental values, and they are suppressed by the attractive potential originating from the outgoing of the electrons in the intense laser fields. More importantly, our calculations reproduce the experimental results that while the tunneling ionization of $\mathrm{Ni}^{+}$is only slightly suppressed, that of $\mathrm{Ni}^{2+}$ is substantially enhanced over the ADK predictions.

The ADK theory is based on the SAE approximation, which excludes the many-electron response embedded in the TDDFT approach. By comparing the TDDFT potential and the SAE potential from OEP calculations, we identified the induced potential that is due to the many-electron response in the laser field. We found it attractive for all three species under study and it lowers their TI rates. For both $\mathrm{Ni}^{+}$and $\mathrm{Ni}^{2+}, 3 d$ to $4 s$ excitation occurs prior to tunneling. Excitation reduces the barrier and hence increases the TI rates.

Both ions are subject to the attractive induced potential that lowers the TI rate and the excitation that increases the TI rate. The energy separation between $4 s$ and $3 d$ is small for $\mathrm{Ni}^{+}$; therefore, the induced potential has a greater impact than excitation, which results in slightly suppressed TI. For $\mathrm{Ni}^{2+}$ the excitation energy is considerably greater, and the overall effect is that TI is enhanced due to the reduced $I_{p}$ following excitation.

\section{ACKNOWLEDGMENT}

This work is supported by National Science Foundation Award No. PHY-1506441.
[1] L. V. Keldysh, Sov. Phys. JETP 20, 1307 (1965).

[2] E. Goulielmakis, Z.-H. Loh, A. Wirth, R. Santra, N. Rohringer, V. S. Yakovlev, S. Zherebtsov, T. Pfeifer, A. M. Azzeer, M. F. Kling et al., Nature (London) 466, 739 (2010).
[3] A. Ferre, A. E. Boguslavskiy, M. Dagan, V. Blanchet, B. D. Bruner, F. Burgy, A. Camper, D. Descamps, B. Fabre, N. Fedorov et al., Nat. Commun. 6, 5952 (2015). 
[4] B. Wolter, M. G. Pullen, A. T. Le, M. Baudisch, K. DoblhoffDier, A. Senftleben, M. Hemmer, C. D. Schroeter, J. Ullrich, T. Pfeifer et al., Science 354, 308 (2016).

[5] Y. Kobayashi, M. Reduzzi, K. F. Chang, H. Timmers, D. M. Neumark, and S. R. Leone, Phys. Rev. Lett. 120, 233201 (2018).

[6] M. V. Ammosov, N. B. Delone, and V. P. Kralnov, Zh. Eksp. Teor. Fiz. 91, 2008 (1986) [Sov. Phys. JETP 64, 1191 (1986)].

[7] S. Augst, D. D. Meyerhofer, D. Strickland, and S. L. Chin, J. Opt. Soc. Am. B 8, 858 (1991).

[8] S. M. Hankin, D. M. Villeneuve, P. B. Corkum, and D. M. Rayner, Phys. Rev. Lett. 84, 5082 (2000).

[9] M. J. DeWitt, E. Wells, and R. R. Jones, Phys. Rev. Lett. 87, 153001 (2001).

[10] M. Smits, C. A. de Lange, A. Stolow, and D. M. Rayner, Phys. Rev. Lett. 93, 213003 (2004).

[11] M. Lezius, V. Blanchet, M. Y. Ivanov, and A. Stolow, J. Chem. Phys. 117, 1575 (2004).

[12] T. Brabec, M. Cote, P. Boulanger, and L. Ramunno, Phys. Rev. Lett. 95, 073001 (2005).

[13] Z. X. Zhao and T. Brabec, J. Phys. B 39, L345 (2006).
[14] Z. Zhao and T. Brabec, J. Mod. Opt. 54, 981 (2007).

[15] X. Chu and G. C. Groenenboom, Phys. Rev. A 94, 053417 (2016).

[16] E. Murakami, R. Mizoguchi, Y. Yoshida, A. Kitashoji, N. Nakashima, and T. Yatsuhashi, J. Photochem. Photobiol. A 369, 16 (2019).

[17] X. Chu and G. C. Groenenboom, Phys. Rev. A 96, 013421 (2017).

[18] E. Runge and E. K. U. Gross, Phys. Rev. Lett. 52, 997 (1984).

[19] E. K. U. Gross and W. Kohn, Adv. Quantum Chem. 21, 255 (1990).

[20] X.-M. Tong and S.-I. Chu, Chem. Phys. 217, 119 (1997).

[21] A. Kramida, Yu. Ralchenko, J. Reader, and NIST ASD Team, NIST Atomic Spectra Database, version 5.3, National Institute of Standards and Technology, Gaithersburg, MD, http://physics. nist.gov/asd.

[22] M. Kalinski, J. H. Eberly, J. A. West, and C. R. Stroud, Phys. Rev. A 67, 032503 (2003).

[23] X. Chu, Phys. Rev. A 82, 023407 (2010). 